Annuaire suisse de politique de développement

25-2 | 2006

Paix et sécurité : les défis lancés à la coopération internationale

\title{
De l'huile sur le feu. La lutte pour les ressources attise l'insatisfaction planétaire
}

\section{Wolfgang Sachs}

\section{CpenEdition}

\section{Journals}

Édition électronique

URL : http://journals.openedition.org/aspd/263

DOI : 10.4000/aspd.263

ISSN : 1663-9669

Éditeur

Institut de hautes études internationales et du développement

Édition imprimée

Date de publication : 1 octobre 2006

Pagination : 129-138

ISBN : 2-88247-064-9

ISSN : $1660-5934$

Référence électronique

Wolfgang Sachs, «De l'huile sur le feu. La lutte pour les ressources attise l'insatisfaction planétaire », Annuaire suisse de politique de développement [En ligne], 25-2 | 2006, mis en ligne le 18 décembre 2009, consulté le 08 septembre 2020. URL : http://journals.openedition.org/aspd/263 ; DOI : https://doi.org/ $10.4000 /$ aspd.263 


\title{
De l'huile sur le feu
}

\author{
La lutte pour les ressources attise l'insatisfaction planétaire
}

Wolfgang Sachs*

\begin{abstract}
- n 1928, Mohandas Gandhi formula l'une de ces intuitions qui ont porté sa pensée jusqu'au XXI ${ }^{\mathrm{e}}$ siècle: «Dieu fasse que l'Inde ne s'industrialise jamais sur le modèle occidental. L'impérialisme économique d'un seul et minuscule royaume insulaire (l'Angleterre) tient actuellement le monde dans ses chaînes. Si une nation comptant 300 millions d'habitants se lançait dans pareille exploitation économique, le monde serait ravagé comme par une invasion de criquets. ${ }^{1}$ Près de quatre-vingts ans plus tard, cette observation résonne de manière effrayante car, entre-temps, ils ne sont plus 300 millions mais 1000 millions à suivre l'exemple de l'Angleterre. Gandhi craignait que la multiplication des Angleterres ne se traduise par l'extension de l'exploitation coloniale, et cela jusqu'à épuisement de la biosphère. Les limites biophysiques à l'extension de la civilisation industrielle à toute la planète sont maintenant clairement visibles; elles confirment de manière impressionnante l'intuition de Gandhi.
\end{abstract}

Depuis que les limites biophysiques de la croissance classique se manifestent, plus rien n'est comme avant, en particulier dans la politique Nord-Sud. La politique part pourtant depuis longtemps du principe que l'égalité sociale sera assurée par la croissance nationale et internationale. L'attelage de l'équité à la croissance a été, dès après la Seconde Guerre mondiale, l'une des pierres angulaires de l'âge du développement. Mais depuis que l'on entrevoit la finitude de la biosphère, soit depuis quelques décennies, cette pierre angulaire se trouve déstabilisée. Simplement parce que, dans un monde limité, la croissance classique ne saurait apporter l'égalité, à moins d'anéantir la biosphère.

Les conflits liés aux ressources et à l'environnement du XXI ${ }^{\mathrm{e}}$ siècle ont donc leur signature: la contradiction entre une demande illimitée en biens naturels et, d'autre part, la finitude de l'environnement. On sait que ces conflits n'ont rien de nouveau; que l'on pense aux luttes entre riverains d'un même cours d'eau au Moyen Age ou, à l'époque de Bismarck, aux démêlés entre nations européennes pour le contrôle des richesses naturelles de l'Afrique, sous le signe de la convoitise, de la pénurie et des rivalités. Entre-temps, le développement a créé une attente universelle et, parallèlement, on a pris conscience des limites de la biosphère. Les conflits pour le contrôle des ressources présentent un nouveau caractère: ils révèlent la contradiction fondamentale entre expansion économique et contraintes écologiques.

Cette contradiction ne se manifeste pas uniquement par des crises environnementales, mais aussi par des crises sociales. La convoitise et la pénurie suscitent

* Directeur de recherche au Wuppertal Institut für Klima, Umwelt, Energie, à Wuppertal (Allemagne) ; professeur invité à l'Université de Kassel (Allemagne) et au Schumacher College, à Darlington (Angleterre).

1 Dans Young India, 20 December 1928 (citation originale en anglais - N.D.E.). 
toujours les rivalités. On sait que les nations et les humains ne profitent pas de la planète de la même manière. La règle empirique reste valable: un quart de la population mondiale s'approprie trois quarts des ressources mondiales. Ce sont avant tout les pays industrialisés qui puisent de manière excessive dans l'héritage naturel et exploitent l'environnement bien au-delà de leurs frontières nationales. Leur empreinte écologique dépasse leur propre territoire - parfois très largement - si l'on comptabilise toutes les ressources et décharges intégrées à leur fonctionnement. Et plus les ressources déclinent, plus la question de leur répartition se fait urgente. A qui appartiennent les réserves de pétrole, les cours d'eau, les forêts, l'atmosphère? Qui a des droits, et lesquels, sur les mécanismes vitaux de la biosphère? Combien chacun peut-il puiser pour son bien-être sans empiéter sur le droit des autres? Ce sont des questions sous-jacentes à bien des conflits pour les ressources ou pour l'environnement.

Les conflits de ce genre sont gros d'un danger, celui que les inégalités ne s'en trouvent que multipliées. En effet, les rivalités qui s'exacerbent dans un contexte de finitude entraînent de nouvelles polarisations sociales. Les puissants s'emparent des ressources encore disponibles et les impuissants en sont pour leurs frais. L'essence augmente pour les pendulaires, les cours d'eau sont asséchés avant terme, le prix des importations de céréales s'envole, les pêcheurs reviennent les filets vides. Les conflits sociaux éclatent bien avant la fin écologique des ressources. Plus l'on s'approche de la limite de portance des écosystèmes, plus la pression s'accroît sur les faibles: les limites écologiques projettent loin devant elles leur ombre socioéconomique, et bien avant d'avoir été définitivement franchies. Avant même que la paix environnementale (au sens écologique du terme) n'ait été sérieusement perturbée, on constate que la paix sociale se fissure.

On constate que de nombreux conflits dont la religion, les luttes de clans, voire l'instauration de la démocratie sont la raison déclarée ont pour cause réelle le partage inéquitable de ressources. La lutte pour les ressources est souvent liée aux conflits de nature politique ou ethnique. Si l'on ignore la question du pétrole, la crise permanente du Proche-Orient et la guerre civile du Soudan deviennent incompréhensibles. Et à ignorer la dégradation et la pénurie des terres, on ne comprend ni la situation des réfugiés au Pakistan ni le génocide du Rwanda. Que ce soit au niveau international ou subnational, les conflits autour des ressources contribuent à la déstabilisation sociale lorsque manquent les procédures d'arbitrage légitimes. Il est donc prévisible qu'en cas de tensions supplémentaires relatives aux ressources, les risques de conflits ne feront que s'aggraver en de nombreux endroits, rendant la Terre encore plus explosive. Nous utilisons ici l'exemple du pétrole pour étudier la manière dont les frontières écologiques - qui représentent bien plus qu'un sujet de recherche biophysique - peuvent provoquer des conflits sociaux, parce qu'on retrouve l' «or noir» dans toutes les constellations conflictuelles types liées à des conflits de ressources: $1^{\circ}$ son extraction conjure des conflits de survie; $2^{\circ}$ sa valorisation économique provoque entre autres des conflits de régime à l'intérieur des pays; $3^{\circ}$ son appropriation par les nations provoque depuis longtemps des conflits de répartition; et $4^{\circ}$ sa combustion engendre des effets qui accentuent les conflits de développement. 
Depuis l'époque de Pizarro, on a systématiquement prospecté le «Nouveau Monde» à la recherche de matières premières précieuses. Aujourd'hui, la frontier de la prospection et de l'exploitation des matières premières a été repoussée aux confins des continents et loin dans les mers, les gisements facilement exploitables ayant déjà été mis en valeur ou étant déjà taris. On extrait du pétrole au cœur de la forêt vierge et au fond des mers. On récolte du bois en Patagonie et en Sibérie, des fabriques flottantes ratissent les mers depuis le cercle polaire arctique jusqu'en Antarctique. Nous sommes particulièrement avides de ressources énergétiques, plus particulièrement le pétrole et le gaz, après quoi viennent les métaux, or, zinc, argent, cobalt, et les matières premières biotiques telles que le bois et les poissons. Depuis l'ouverture des frontières aux entreprises transnationales dans le sillage de la globalisation, une formidable pression repousse sans cesse les limites de l'exploitation. L'industrie extractive progresse désormais dans des terrains jusqu'alors hors jeu, en général des territoires de sociétés indigènes, et les intègre au réseau mondial des flux de ressources.

C'est en particulier le cas des territoires amazoniens de l'Equateur ${ }^{2}$. Depuis le premier forage du consortium Texaco-Gulf, en 1964, l'Oriente, comme on le nomme, vit à l'âge du pétrole. Au cours de ces trente dernières années, les compagnies pétrolières se sont lancées les unes après les autres sur ce territoire représentant le tiers du pays, y ont érigé leurs tours de forage et installé une infrastructure d'exploitation aux ramifications lointaines. Le pétrole apporte un bon lot de devises à l'Etat, des dollars. Le budget national n'en souffre pas moins d'un déficit chronique - comme dans de nombreux Etats d'Amérique latine - et l'Etat s'est endetté à l'étranger. L'exploitation du pétrole semblait devoir tirer le pays d'affaire avec ses rentrées programmées et ses exportations synonymes de devises. Le pétrole est généralement exporté aux Etats-Unis. Les concessions accordées sans discernement aux groupes multinationaux (entre autres Agip, Mobil, Amoco, Elf Aquitaine, Petrobras, Texaco) couvrent 1,2 million d'hectares de forêt tropicale (sur 13 millions) et correspondent souvent à des territoires indigènes.

Les peuples vivant en Oriente sont principalement des sociétés de chasseurscueilleurs, de langues et traditions diverses. Il s'agit en premier lieu des Quichua, des Huaorani et des Shuar. L'ensemble des groupes indigènes représente environ $125^{\prime} 000$ personnes sur un territoire à faible densité de population. La conjonction forêt-eau fait de cette région l'une des plus riches en espèces du monde et les groupes indigènes dépendent entièrement des espaces naturels de forêts, des zones inondées et des berges des cours d'eau.

L'exploitation du pétrole n'a rien de discret: explosions, installations de pompage, pipelines, raffineries, tranchées dans la forêt, routes, pistes, véhicules lourds et campements. En Oriente, la première mesure fut généralement de déboiser, et la surface forestière ne représente plus que $30 \%$ de celle des années 1970; la surface appartenant aux communautés indigènes s'est réduite à $8 \%$. Une torchère brûle en continu les restes de pétrole et de gaz, on taille des tranchées et fore à la dynamite sans égard pour les lieux sacrés des Indios. La 
pollution de l'eau a rapidement atteint un niveau tragique: les rejets et déchets empoisonnés polluent les ruisseaux et les rivières où les habitants puisent leur eau de consommation. De nombreuses fuites et ruptures de tuyaux laissent le pétrole s'infiltrer dans le sol et l'eau. On estime qu'au cours des vingt dernières années, une trentaine de ruptures de pipelines ont laissé un demi-million de barils de pétrole s'échapper dans les cours d'eau équatoriens. Le déboisement et l'empoisonnement ont entrainé la disparition de plantes, de poissons et d'animaux sauvages, réduisant la base vitale des groupes indigènes. Les conséquences sont la malnutrition, la désintégration sociale et, finalement, l'expulsion.

L'exemple de l'Equateur illustre un processus récurrent. D'un côté, la demande mondiale en ressources naturelles de toutes sortes augmente; de l'autre, ces ressources n'attendent pas simplement quelque part en terrain neutre qu'on vienne les chercher. Au contraire, elles se trouvent bien souvent sur le territoire d'indigènes et constituent une des bases de leur vie. L'exploitation non réglementée des ressources peut dégrader, décimer, voire désacraliser leurs paysages, en particulier dans les périphéries du Sud. L'exploitation mesurée de l'écosystème vu comme un bien commun est battue en brèche par une exploitation ne visant qu'à maximiser les revenus d'un investissement ${ }^{3}$. Les besoins sanitaires des communautés locales et, tout bonnement, leur survie se trouvent marginalisés par rapport aux besoins en carburant et combustibles de consommateurs lointains. La subsistance des unes se trouve en concurrence avec les goûts de luxe des autres. C'est parce que l'exploitation pétrolière, la déforestation et la pêche industrielle menacent les ressources vitales des habitants qu'elles provoquent leur résistance. Les pauvres sont en quelque sorte dépossédés de leurs ressources pour que les riches puissent vivre au-dessus de leurs moyens.

Les conflits de régime

Naguère encore, la découverte de champs pétrolifères suscitait l'enthousiasme. Les théories habituelles du développement y voyaient le gage de l'accession d'un pays au bien-être et à la démocratie. Mais un coup d'œil sur la réalité et les études empiriques relativisent cette image idéalisée. Les études comparatives montrent que, en fait, les pays riches en ressources ont une croissance moindre que les autres et disposent d'un revenu inférieur par habitant. De plus, 12 des 25 pays du monde qui dépendent le plus de leurs ressources en matières premières et 6 des pays qui dépendent le plus de leur pétrole se trouvent dans le groupe des 25 pays les plus endettés. Plus un pays dépend de l'exportation de ses trésors naturels, moins son indice de développement humain est élevé. En ce qui concerne la mortalité infantile, l'espérance de vie et l'éducation, elles sont généralement à la traîne de la moyenne des pays moins riches en ressources. D'où la thèse de la «malédiction des ressources » qui a remplacé celle de la «bénédiction des ressources $»^{4}$.

3 W. Sachs, Ökologie und Menschenrechte, Wuppertal Paper, $n^{\circ}$ 131, Wuppertal, Wuppertal Institut für Klima, Umwelt, Energie, 2003.

4 Pour un résumé: M. Renner, The Anatomy of Resource Wars, Worldwatch Paper, $\mathrm{n}^{\circ}$ 162, Washington, Worldwatch Institute, 2002. P. Le Billon, «The Geopolitical Economy of "Resource Wars"», dans Geopolitics of Resource Wars: Resource Dependence, Governance and Violence, P. Le Billon (ed.), London, Frank Cass, 2005, pp. 1-28. 
En Iran, en Russie et dans certains pays du monde arabe ou de l'Afrique, la malédiction est accablante. Au Nigeria, par exemple: «Il maudit la terre qui crachait le pétrole, crachait l'or noir. Il maudit les Dieux qui n'avaient pas asséché les puits $»^{5}$, écrivait l'auteur nigérian Ken Saro-Wiwa dans sa nouvelle Night Ride. En 1995, Ken Saro-Wiwa fut exécuté pour avoir mobilisé l'opinion publique contre les ravages commis par le groupe Shell en pays ogoni avec la complicité du monde politique nigérian. Alors que, suivant des chemins détournées, les revenus pétroliers continuent d'affluer dans les poches des groupes dominants, les installations pétrolières du delta du Niger sont régulièrement la cible des chantages de groupes violents. Un Etat en déliquescence, où des bandes de maraudeurs mettent les pipelines en perce et une caste corrompue emplit ses comptes à l'étranger: le Nigeria est l'exemple du pays victime de son pétrole. D'autres pays riches en hydrocarbures tels que l'Angola, le Cameroun ou le Soudan sont tout aussi opaques et répressifs que les Etats pétroliers bien établis: Arabie saoudite, Koweït, Irak, Brunei, Russie... dont on peut tout dire sauf qu'ils illustrent la démocratie.

Cette situation n'a rien de surprenant. Les Etats dont le revenu dépend prioritairement des redevances sur l'exploitation de leurs ressources et non pas des impôts versés par leurs habitants glissent facilement dans une culture de la corruption. Seuls les pays dotés d'un régime bien ancré et placé sous contrôle public semblent échapper à ce destin. Ainsi, ni le Mexique ni la Norvège n'ont les traits d'un Etat rentier. On parle d'Etat rentier lorsque les détenteurs du pouvoir peuvent se permettre de ne plus se sentir de devoirs envers la population parce que leurs revenus croissent sans effort et semblent illimités. Ceux qui se sont emparés du pouvoir disposent de moyens suffisants pour instaurer un régime de favoritisme qui récompense les sujets dociles et harcèle les opposants. L'Etat n'a alors plus de raison de rendre de comptes à la population et bloque tout partage politique. Les élites dominantes adoptent une mentalité de rentiers portés sur la dépense et la consommation, sans se soucier de doter le pays d'une force de production. Ce genre de régime doit régulièrement recourir à la force pour se maintenir au pouvoir, ce qui provoque souvent des réactions violentes et, en tout cas, est cause d'insatisfaction chronique.

Tous ces conflits semblent bien éloignés des colonnes à essence, des citernes de mazout et de l'air conditionné des parties riches du monde. Et pourtant le pétrole - tout comme les diamants, le coltan, le cuivre ou les bois précieux finit en général par être consommé dans les pays riches du Nord et du Sud, même lorsque son cheminement est difficile à reconstituer. En dernière analyse, c'est la demande des pays riches qui rend l'exploitation des ressources si lucrative que des régimes kleptocrates peuvent en vivre. Et c'est le besoin de voies de ravitaillement fiables qui entraîne les gouvernements et les entreprises des pays démocratiques dans la complicité avec les régimes autoritaires que sont, par exemple, l'Arabie saoudite et, toujours plus, la Russie. Par ailleurs, plus la valeur de la ressource pétrole augmente, plus on a lieu de craindre qu'elle attire les activités criminelles. Il n'est sans doute pas exagéré de voir que là aussi, il y a bien des similitudes entre la dépendance aux drogues et celle au pétrole.

5 K. Saro-Wiwa, «Night Ride», dans A Forest of Flowers: Short Stories, Port Harcourt (Nigeria), Saros International Publishers, 1986, p. 114 (citation originale en anglais - N.D.E.). 
Le pétrole est actuellement la plus importante ressource planétaire, plus que l'or ne l'a jamais été. Sans pétrole, le système industriel et économique s'effondrerait: l'industrie et l'emploi reposent largement sur l'utilisation ou la transformation du pétrole brut; la mobilité et les transports sur l'eau, sur terre et dans les airs dépendent principalement des produits pétroliers; il en va de même du plastique, des médicaments, des engrais, des matériaux de construction, des couleurs, des textiles et de bien d'autres choses. La dépendance au pétrole n'a fait qu'augmenter depuis le milieu du siècle dernier. Le pétrole est devenu une ressource politique, économique et même culturelle irremplaçable. Plus que tout autre produit, il détermine le style de vie des habitants de la planète ${ }^{6}$.

La sécurisation de l'accès aux hydrocarbures est depuis longtemps l'un des facteurs centraux de la géopolitique. Les Etats orientent leurs politiques économique, étrangère et militaire de manière à accroître leur influence sur les ressources importantes localisées en d'autres régions du monde. Il est patent que les Etats-Unis et leurs alliés n'ont pas lancé leurs machines de guerre uniquement pour libérer le Koweït, provoquer la chute de la dictature en Irak ou anéantir le réseau terroriste d'Al-Qaida, mais aussi pour consolider leur contrôle sur les réserves de pétrole du Moyen-Orient. Car le pétrole, qui a pris tant d'importance dans les pays industrialisés, n'est souvent pas disponible là où on le consomme. C'est pourquoi l'accès aux sources étrangères présente une telle priorité que les efforts déployés pour le garantir ont, ces dernières décennies, plusieurs fois dégénéré en conflits généralisés et en guerres meurtrières. Il y a aujourd'hui un siècle que les nations se disputent le partage des réserves de pétrole pour asseoir leur puissance et développer leur économie.

L'importance géopolitique du pétrole a sensiblement augmenté depuis que la fin des réserves est en vue. Il y a quelques décennies encore, les exportateurs se félicitaient régulièrement de la constante augmentation des réserves, qui était supérieure à celle de la consommation. La situation s'est inversée au début des années 1980: l'exploitation annuelle dépasse de beaucoup les nouvelles découvertes. Pour chaque baril de pétrole nouvellement découvert, on en pompe actuellement quatre. Si l'on considère l'ensemble des champs pétrolifères du monde, on peut déterminer le «point d'extraction maximale», dit Depletion Mid Point, après lequel la production baissera irrémédiablement. Selon toute vraisemblance, ce point sera atteint entre 2008 et $2015^{7}$.

Dans un tel contexte, les stratégies géopolitiques visant à garantir le ravitaillement en pétrole et à assurer la stabilité de son prix prennent une importance particulière. Dès que le point d'extraction maximale sera atteint, le prix des hydrocarbures s'envolera, risquant de provoquer un cercle vicieux semblable à celui de la crise pétrolière des années 1970, à la différence près qu'il n'y aura pas de fin de la pénurie en vue. Lorsque les quantités produites ne pourront plus satis-

6 Wuppertal Institut, Fair Future. Begrenzte Ressourcen und globale Gerechtigkeit, München, C.H. Beck Verlag, 2005.

7 C.J. Campbell, The Coming Oil Crisis, Brentwood, uK, Multi-Science Publishing Compagny; Petroconsultants SA, 2004. P. Hennicke, M. Müller, Weltmacht Energie. Herausforderung für Demokratie und Wohlstand, Stuttgart, Hirzel, 2005. 
faire la demande, tous les pays, en particulier ceux qui dépendent d'importations de pétrole, subiront une crise économique.

Cette crise s'annonce alors que le monde entier se montre toujours plus assoiffé de pétrole. Selon toutes les prévisions, la consommation devrait augmenter spectaculairement. A la demande des pays déjà bien industrialisés s'ajoute depuis quelque temps celle des nouveaux consommateurs, en particulier la Chine, l'Inde et le Brésil. Leur besoin en pétrole - matière première et combustible à ce jour irremplaçable - croît rapidement, ce qui en fait de gros acheteurs sur le marché mondial. Ce sont particulièrement les pays seuils d'Asie, dans lesquels les réserves de gaz et de pétrole sont relativement limitées, qui attisent la concurrence. La Chine est déjà le second importateur mondial de pétrole et les pays tels que la Malaisie, le Vietnam ou l'Indonésie, aujourd'hui encore exportateurs, vont se transformer en importateurs nets dans la décennie. Comme les pays industrialisés sont loin de réduire leur consommation de manière importante (en fait, beaucoup d'entre eux l'augmentent!), la situation se tend. Les pays en pleine croissance font connaître leurs exigences, les anciens consommateurs n'entendent pas lever le pied, et les réserves s'amenuisent: les rivalités sont là, le scénario des conflits des prochaines décennies est tout tracé.

Le gâteau diminue, l'appétit et le nombre des hôtes augmentent: le conflit est programmé et s'annonce durable ${ }^{8}$. Les batailles de demain projettent déjà leur ombre sur le Golfe, l'Afghanistan, la mer Caspienne et la Tchétchénie. Ce sont les pays pauvres qui paieront le prix fort. Les analyses de l'Agence internationale de l'énergie chiffrent les premiers effets d'une augmentation de 15 à 25 dollars le baril dans les pays industrialisés à une réduction de la croissance de $0,4 \%$ alors que les pays en développement importateurs de pétrole seront touchés beaucoup plus durement, à hauteur de $0,8 \%$ pour les pays asiatiques et jusqu'à $3,0 \%$ pour les pays du sud du Sahara'. L'endettement de ces pays crốtra, comme ce fut le cas durant la crise pétrolière de 1973, et toute une part de la population ne pourra plus s'offrir d'énergie fossile. La croissance économique s'en trouvera enrayée et, comme c'est déjà le cas dans beaucoup d'Etats africains où l'essence manque, les véhicules utilitaires resteront à l'arrêt. Les entreprises qui pourront tenir pendant l'envolée du coût de l'énergie verront leurs concurrents disparaître. De bien sombres perspectives pour les pauvres: il est probable que l'évolution du prix du pétrole enfoncera les pays à moindre pouvoir d'achat plus profond dans l'apartheid économique. Au lieu de diminuer, les inégalités ne feront qu'augmenter.

Les limites sociales de la consommation de pétrole seront donc visibles bien avant les limites écologiques. La finitude des réserves de pétrole sera un facteur de déstabilisation bien avant que le dernier baril ne soit pompé. Les «limites de la croissance» reviennent sous forme de conflit géopolitique. Dans ce contexte, il est à peine exagéré d'affirmer que le développement économique conventionnel, qui se nourrit d'énergies fossiles, est devenu un gros facteur de risque pour la sécurité mondiale. Voilà qui renverse l'une des évidences chéries de la

\footnotetext{
Voir la contribution de N. Supersberger dans P. Hennicke, M. Müller, op. cit.

9 International Energy Agency (IEA), Analysis of the Impact of High Oil Prices on the Global Economy, Paris, 2004.
} 
seconde moitié du $\mathrm{Xx}^{\mathrm{e}}$ siècle: le développement ne favorise par la paix; au contraire, tant qu'il s'appuiera sur le pétrole, le gaz ou le charbon, il sera un facteur de conflits.

\section{Les conflits de développement}

Ce n'est pas uniquement parce qu'ils seront écartés de la course aux ressources fossiles que les pays pauvres se trouveront sous pression, mais aussi parce qu'ils auront beaucoup à souffrir des effets nocifs de la combustion du pétrole. Selon toute probabilité, ce sont les pays et les personnes qui auront le moins contribué au changement climatique qui, les premiers, en goûteront les effets amers. A l'inverse de ce que tend à faire accroire le film The Day after Tomorrow ${ }^{10}$, le changement ne sera pas instantané et catastrophique, mais commencera de manière peu visible et sournoise. Et les pays touchés ne seront pas ceux d'Amérique du Nord ou d'Europe, mais bien plus ceux de l'hémisphère Sud. Les grands deltas de Chine, du Vietnam, du Nigeria et, en particulier, du Bangladesh, les petits Etats insulaires des mers du Sud, les domaines arides ou semiarides du globe entier... voilà qui seront les doubles perdants, premièrement parce qu'ils ont moins de ressources transformables en bien-être et deuxièmement parce qu'ils seront les premiers à supporter les conséquences du changement climatique: sécheresses, orages, manque d'eau, élévation du niveau des mers et recul des récoltes. Loin de n'être qu'un sujet de préoccupation pour écologistes, le changement climatique sera très certainement la main invisible qui précipitera le déclin de l'agriculture, l'érosion sociale et l'exclusion.

La destruction coloniale se joue cette fois sans puissance impériale et sans troupe d'occupation. Elle se propage dans l'air, invisible, perfide, télétransportée par la chimie de l'atmosphère. Lorsque l'atmosphère terrestre se réchauffe, la nature se fait instable. On ne pourra progressivement plus de fier aux pluies, aux nappes phréatiques, à la température, au vent ou aux saisons, autant de facteurs qui, depuis des temps immémoriaux, ont assuré aux plantes, aux animaux et aux humains un environnement hospitalier. Avec les perturbations climatiques, bien des régions deviendront moins hospitalières, parfois même inhabitables pour certaines espèces d'animaux et de plantes, voire pour les humains. Il n'est pas difficile de comprendre que l'élévation du niveau des mers rendra inhabitables certaines des terres les plus densément peuplées. Il est aussi clair, bien qu'on y pense moins, que les changements d'humidité et de température provoqueront des modifications dans la végétation et dans la diversité, dans la fertilité des sols et dans les réserves d'eau. Il faut également s'attendre à ce que l'environnement soit moins salubre. Les récoltes seront décimées par les parasites et les mauvaises herbes pendant que les humains seront plus nombreux à être frappés par la malaria, la dengue ou des maladies infectieuses ${ }^{11}$. Les recherches indiquent qu'une augmentation globale de la température de 2 degrés d'ici à 2050 (ce qui nous attend si nous ne réduisons pas les émissions) menacera 25 millions de personnes d'inondations côtières,

10 Film de Roland Emmerich, sorti en 2004.

11 Intergovernmental Panel on Climate Change (IPCC) (ed.), Climate Change 2001 : Impacts, Adaptation, and Vulnerability, contribution of Working Group II to the Third Assessment Report of the IPCC, Cambridge, Cambridge University Press, 2001. 
180 à 250 millions de personnes de malaria et 200 à 300 millions de personnes de manque d'eau ${ }^{12}$.

Les effets nocifs du changement climatique n'atteignent pas tout le monde de la même manière. Les études sur la question le confirment ${ }^{13}$ : les pays du Sud et plus particulièrement leurs habitants au pouvoir d'achat le plus réduit, ceux qui dépendent directement de la nature, sentiront les effets déstabilisants du réchauffement de la planète de manière plus rude que les pays industrialisés et les populations urbaines. Alors que le changement climatique est avant tout dû à la classe minoritaire des consommateurs transnationaux, c'est en grande partie le reste du monde qui paiera la facture. Un nouvel exemple: aujourd'hui déjà, les Inuit (Eskimo), qui vivent dans la région arctique du Canada, sont économiquement et culturellement déstabilisés par l'augmentation de la température. Des chasseurs disparaissent en cours d'expédition parce que, sur leurs cheminements habituels, la glace n'est plus assez portante; les réserves pourrissent parce que le permafrost fond; les igloos perdent leur effet isolant lorsque la neige fond puis gèle à nouveau. Et le dégel des rives entraîne le rejet d'eau douce, poissons compris, dans l'océan Arctique.

C'est pour les plus vulnérables que le danger est le plus grand. En l'occurrence, les riziculteurs du delta du Mékong et les pêcheurs de la côte du Sénégal, les éleveurs du haut pays éthiopien et les habitants des bidonvilles des coteaux de La Paz. Les bases économiques d'innombrables villages et villes seront touchées par les changements dans la production et la productivité agricoles. L'exode rural pourrait augmenter. Les quartiers miséreux seront exposés aux coulées de boue et à la destruction. La maladie ira frapper les moins résistants, les pauvres. Les risques découlant du réchauffement planétaire ne sont en aucun cas équitablement répartis sur la population mondiale; ils pèsent surproportionnellement sur les couches sociales faibles et les dépossédés qui vivent déjà dans des quartiers misérables, dans les régions marginales, et avec le minimum existentiel. Et ce ne sont pas eux qui, par une consommation excessive de combustible dans un contexte de bien-être, sapent leur espace vital.

\section{Les politiques de l'environnement sont des politiques de paix}

Les conflits pour les ressources sont le combustible des petites et grandes confrontations. Ils enflamment des villages et des pays entiers. Nous avons vu à quel point l'histoire récente du Proche-Orient et des autres régions en guerre est gorgée de pétrole; à quel point les conflits religieux et ethniques sont provoqués par le manque de terrain ou d'eau. L'actualité économique nous montre à quel point les pays très peuplés tels que la Chine et l'Inde vont chercher le pétrole et le cuivre, le soja et le bois au-delà de leurs frontières. En résumé, l'appétit pour les ressources prive le monde de la paix. On ne pourra pas assurer l'ordre et la

12 M. Parry et al., «Millions at Risk: Defining Critical Climate Change Threats and Targets », Global Environmental Change, vol. 11, $\mathrm{n}^{\circ} 3,2001$, pp. 181-183.

13 W. Hare, Assessment of Knowledge on Impacts of Climate Change: Contribution to the Specification of Art. 2 of the UNFCCC, Externe Expertise für das WBGU-Sondergutachten «Welt im Wandel: Über Kioto hinausdenken. Klimaschutzstrategien für das 21. Jahrhundert», Berlin, Wissenschaftlicher Beirat der Bundesregierung Globale Umweltveränderungen (WBGU), 2003. 
sécurité globale tant que l'on n'abordera pas les biens de la nature avec plus de respect. Si l'on préfère la sécurité à la déstabilisation et à la violence, il est aujourd'hui nécessaire de réduire nos prétentions sur la biosphère. Les stratégies pour une meilleure utilisation des ressources sont donc aussi des politiques de paix.

Il serait toutefois erroné de n'étudier les conflits pour les ressources que sous l'angle de la sécurité. Finalement, il s'agit de justice ou d'injustice, de puissance ou d'impuissance; il s'agit du respect de l'équité dans l'espace transnational. Le fait que la tendance actuelle rapproche les questions d'environnement et de sécurité est dangereux. Parce que ceux qui parlent de sécurité ne pensent en général qu'à leur propre sécurité, pas à celle des autres. C'est pourquoi il faut bien voir dans les conflits qui agitent la planète non seulement une question de sécurité, mais aussi le résultat d'injustices. La sagesse politique le sait, qui l'a formulé jadis sous forme de maxime: «La paix est l'œuvre de la justice.» 